\title{
Determination of the ODF of Hexagonal Symmetry Materials According to the Maximum Entropy Method
}

\author{
WANG FU, XU JIAZHENG and LIANG ZHIDE \\ Department of Materials Science and Engineering, Northeast University of \\ Technology, Sheyang, Liaoning, China
}

(Received August 1, 1988; in final form October 5, 1988)

\begin{abstract}
The maximum entropy method (MEM) was applied to the determination of orientation distribution functions (ODF) of hexagonal materials. It shows that the ODF determination may be treated as a standard problem of the MEM. Two examples, which confirm the preferable applicability of this method and the reliability of its results, are presented.
\end{abstract}

KEY WORDS: Series expansion, incomplete ODF, maximum-entropy estimate, Lagrangian multiplier, modified Newton method, average orientation density.

\section{INTRODUCTION}

The ODF expressed in the form of generalized spherical harmonic function series (Bunge 1969; Roe 1965) is an ideal representation for the texture of polycrystalline material. There, the ODF series should possess an infinite number of terms with corresponding coefficients $W_{l m n}$ which fulfilled the requirements of the crystal symmetries and the sample symmetries. However, owing to the limitations of conventional texture measurement technique there are only some $W_{l m n}$ of even $L$ which can be directly derived from the experimental data. So, some assumption must be introduction 
for the practical texture representation. The simplest one is assuming that all the missing $W_{l m n}$ are equal to zero and the series obtained is called the reduced ODF or the incomplete ODF. In order to make the result more approaching to the ideal infinite series (i.e. the complete ODF) much effort has been made for the development of new methods (such as Bunge and Esling, 1981; Pospiech, Lücke and Jura, 1981; Van Houtte, 1982; Dahms and Bunge, 1988).

It was pointed out that the result from data processing should be consistent with known information and maximally noncommittal with regard to the unknowns (Jaynes, 1957). A method developed from the maximum-entropy estimate (Shannon, 1949) meets the needs of that principle. This method has been successfully applied to the crystal structure determination (Wei, 1985), the RDF of non-crystalline materials determination (Wei, 1986) and the inverse Pole figure determination (Wang, Xu and Liang, 1987). In this paper the same method was applied to the ODF determination. For simplicity, hexagonal crystal symmetry materials were selected as samples at first.

\section{PRINCIPLES OF THE MAXIMUM-ENTROPY ESTIMATES}

Suppose that a system could be situated in $J$ discrete states. Let $R$ $(R<J)$ be the number of state functions with known expectation values, $B_{r}$ be the known expectation value of $r$-th state function of the system and $A_{r j}$ be the value of $r$-th state function when the system takes state $j$. How can the probability distribution $P_{j}$ be determined?

From the given conditions one can write

$$
\sum_{j=1}^{J} P_{j}=1
$$

and

$$
\sum_{j=1}^{J} P_{j} A_{r j}=B_{r}, \quad r=1,2, \ldots, R .
$$

In such a case of $R<J$, it seems insufficient to determine the distribution of probabilities $P_{j}$ from equation set (1). However, the 
maximum-entropy estimate has confirmed that: i) there is a unique, unambiquous criterion for the "amount of uncertainty" $H\left(P_{1}\right.$, $\left.P_{2}, \ldots, P_{J}\right)$ represented by a discrete probability distribution which agrees with whatever information is given; ii) $H\left(P_{1}, P_{2}, \ldots, P_{J}\right)$, which has been called the entropy of the probability distribution $P_{j}$, takes the form

$$
H\left(P_{1}, P_{2}, \ldots, P_{J}\right)=-K \sum_{j=1}^{J} P_{j} \ln P_{j},
$$

where $K$ is a positive constant and iii) the probability distribution subject to whatever is known has a maximum entropy. According to this estimate, one can introduce the Lagrangian multiplier method in the usual way for maximizing Eq. (2) under the constraints of equation set (1) and the result has the form

$$
P_{j}=\exp \left[-1-\lambda_{0}-\sum_{r=1}^{R} \lambda_{r} A_{r j}\right], \quad j=1,2, \ldots, J .
$$

where $\lambda_{0}, \lambda_{1}, \lambda_{2}, \ldots, \lambda_{R}$ are the Lagrangian unkown multipliers. Introducing equation set (3) into equation set (1), a set of non-linear equations of the unknown multipliers can be obtained and all the multipliers can be determined. So the problem of finding the probability distribution is solved.

\section{APPLICATION TO THE ODF DETERMINATION}

Although the maximum-entropy estimate is just suitable for the distribution of discrete probabilities, yet it has been successfully applied to solve some problems with continuous distribution (see "Introduction") when the distribution is artificially dispersed into discrete form. Following this thought, divide the whole orientation space represented by Euler angles $(\psi, \theta, \varphi)$ into $J$ orientation elements $(\sin \theta \Delta \theta \Delta \psi \Delta \varphi)$ and let $V_{j}$ be the volume fraction of the sample oriented along element $j$, then their summation is equal to one.

$$
\sum_{j=1}^{J} V_{j}=1
$$


From the definition of orientation density $\omega(\theta, \psi, \varphi)$, it is

$$
V_{j}=\omega\left(\theta_{j}, \psi_{j}, \varphi_{j}\right) \sin \theta_{j} \Delta \psi \Delta \varphi,
$$

where $\omega\left(\theta, \psi_{j}, \varphi_{j}\right)$, is the average orientation density of element $j$. According to the series expansion method, the lmn-th term coefficient of the ODF series, $W_{l m n}$, is expressed by

$$
\begin{aligned}
W_{l m n}= & \frac{1}{4 \pi^{2}} \int_{0}^{2 \pi} \int_{0}^{2 \pi} \int_{0}^{\pi} \omega(\theta, \psi, \varphi) Z_{l m n}(\cos \theta) \\
& \times e^{i m \psi} e^{i n \varphi} \sin \theta d \theta d \psi d \varphi,
\end{aligned}
$$

where $Z_{\operatorname{lmn}}(\cos \theta)$ is an augmented Jacobi polynomials. Substituting summation for integration in Eq. (6), it becomes

$$
W_{l m n}=\frac{1}{4 \pi^{2}} \sum_{j=1}^{J} Z_{l m n}\left(\cos \theta_{j}\right) \exp \left[i\left(m_{\psi j}+n_{\varphi j}\right)\right] V_{j}
$$

Let $P_{j}, \quad B_{r}$ and $A_{r j}$ take the places of $V_{j}, W_{l m n}$ and $\left(1 / 4 \pi^{2}\right) Z_{l m n}\left(\cos \theta_{j}\right) \exp \left[i\left(m \psi_{j}+n \varphi_{j}\right)\right]$ respectively, then Eqs. (4) and (7) become identical with equation set (1). That means, the ODF determination may be treated as a MEM problem also.

\section{EXPERIMENTAL TEST}

The tested samples were hexagonal system materials with symmetry planes perpendicular to each of the four crystal axes and with statistical symmetry planes perpendicular to the three sample coordinate axes. In this situation, $A_{r j}$ takes on the form

$$
\begin{aligned}
A_{r j}= & \frac{1}{8 \pi^{2}}\left[Z_{l m n}\left(\cos \theta_{j}\right) \cos \left(m \psi_{j}+n \varphi_{j}\right)\right. \\
& \left.+(-1)^{L} Z_{l m N}\left(\cos \theta_{j}\right) \cos \left(m \psi_{j}-n \varphi_{j}\right)\right],
\end{aligned}
$$

where $r$ is the order number of the $\operatorname{lm} n$ array and $R$ is the total number of $W_{l m n}$ to be used in processing data. The incomplete ODF series were expanded up to $L=16$ with $R=107$. For the application of MEM, put $J=3888, \Delta \theta=\Delta \psi=\Delta \varphi=5^{\circ}$. The non-linear equation sets for determination of the Lagrangian multiplier of Eq. (3) were solved by the modified Newton method. The resultant $\left\{\omega\left(\theta_{j}\right.\right.$, 
$\left.\left.\psi_{j}, \varphi_{j}\right)\right\}$ of each sample obtained from Eq. (5) was expressed as a set of constant- $\psi$ or constant- $\varphi$ sections respectively. Two examples are presented here.

(a) A presumed sheet sample

The presumed true ODF of a sheet sample shown in Figure 1 is expressed as a set of constant- $\psi$ sections with orientation densities according to the labelled equidensity contours. It should be pointed out that in each section the basic density is equal to zero and the density within any contour is uniform and equal to the density value labelled.

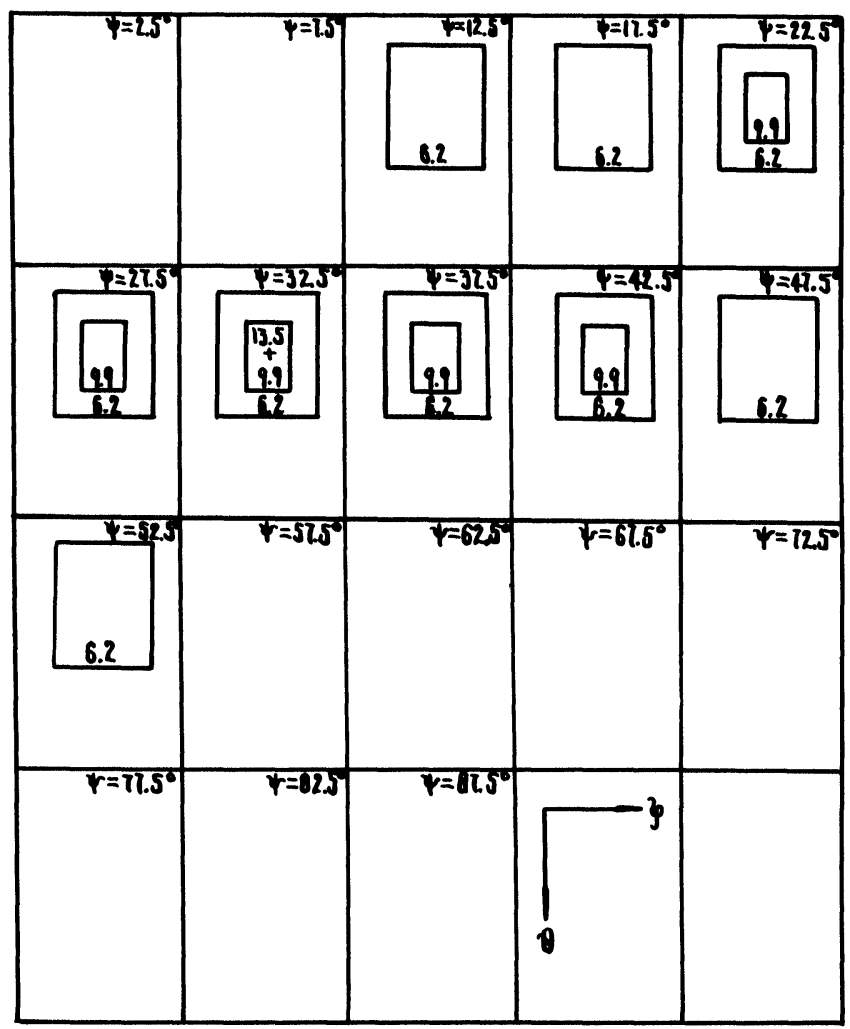

Figure 1 The orientation density distribution of the presumed sample. 


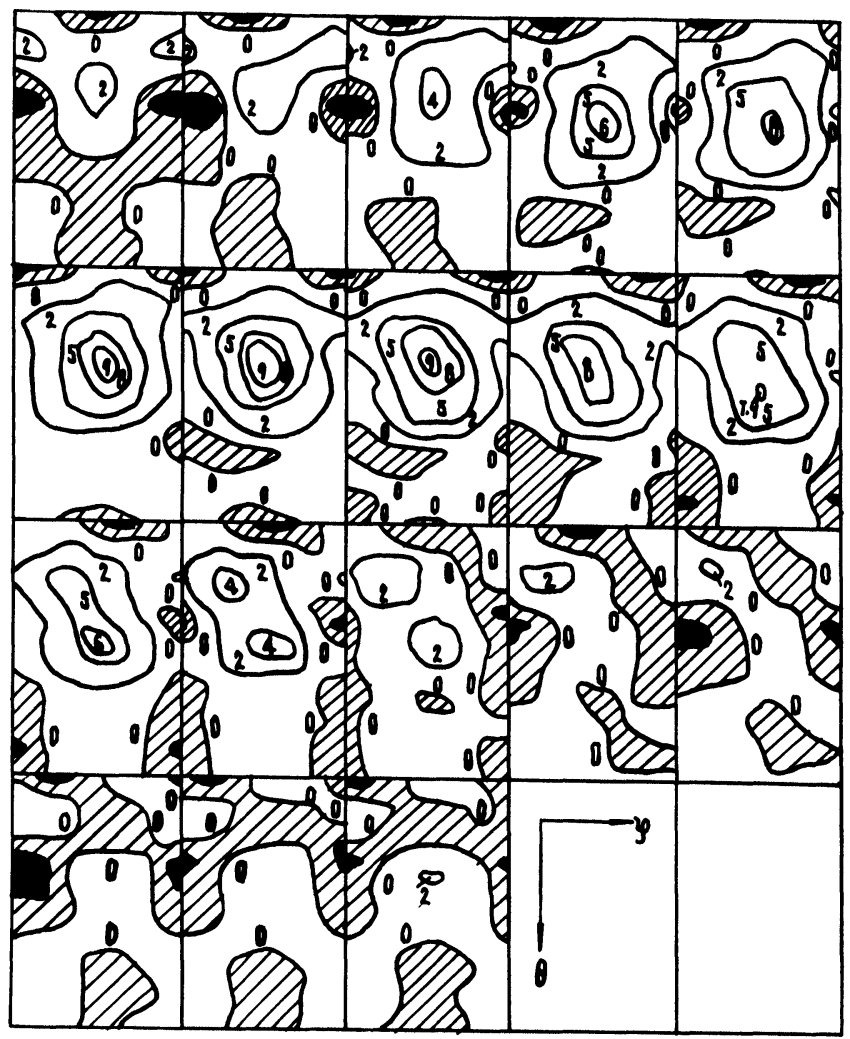

Figure 2 The incomplete ODF of the sample reproduced by the series expansion method with $l_{\max }=16$ (refer to the $\psi$-values being the same as in Figure 1).

Figure 2 is the incomplete ODF of the presumed sample reproduced by the series expansion method. The regions with shadow stripes are the regions in which the density value is negative and the dark regions have density value less than -2 . The configuration of Figure 2 keeps more or less alike that of Figure 1, but the negative regions and the ghost peaks are exhibited markedly.

Figure 3 is the ODF reproduced by the MEM using the same $W_{l m n}$ set as in Figure 2. Here, distortion of the orientation distribution is reduced appositely, negative regions have disap- 


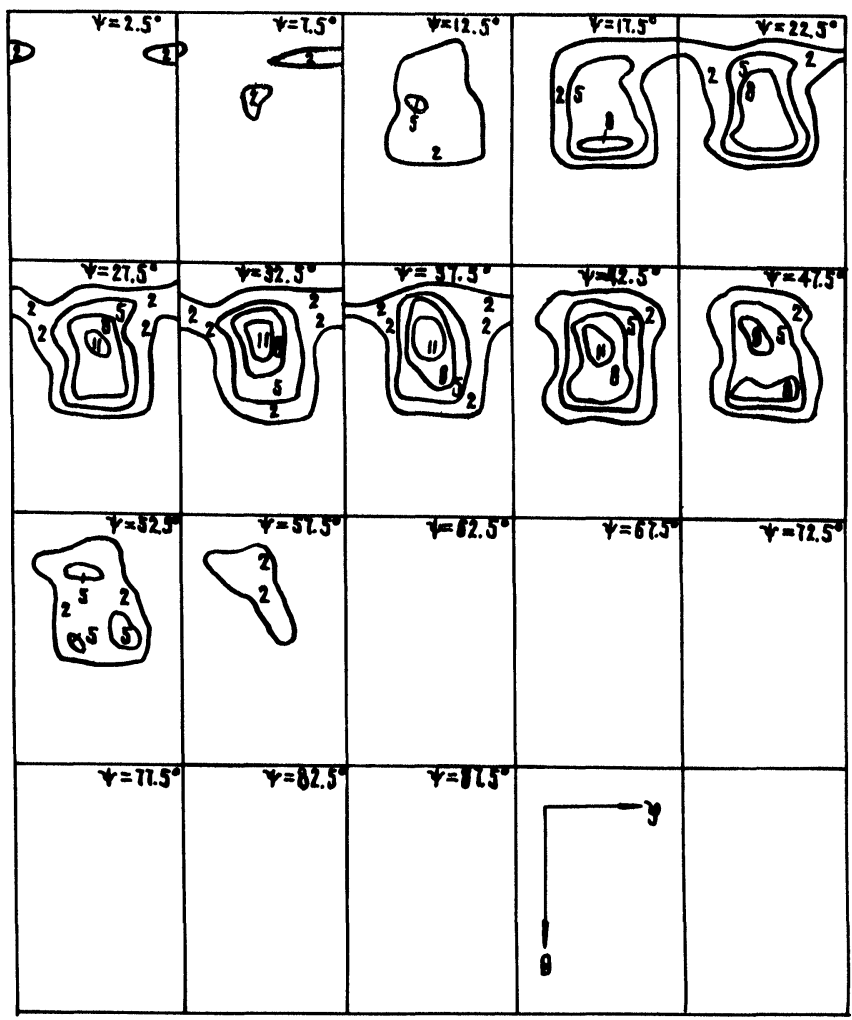

Figure 3 The ODF reproduced by the MEM using the same $W_{l m n}$ set as in Figure 2 .

peared and remnants of the ghosts become very narrow and weak.

Figure 4 is the orientation density versus $\theta$ curves along $\psi=32.5^{\circ}$ and $\varphi=32.5^{\circ}$. It is clear that the curve calculated by the MEM is closer to the originally assumed one. In addition, some odd $L$ term coefficients of the ODF calculated by the MEM are collected in Table 1.

(b) A sample cut from multi-direction rolled $\alpha$-Ti alloy sheet.

The incomplete ODF of this sample determined by the series expansion method from several measured pole figures is shown in 


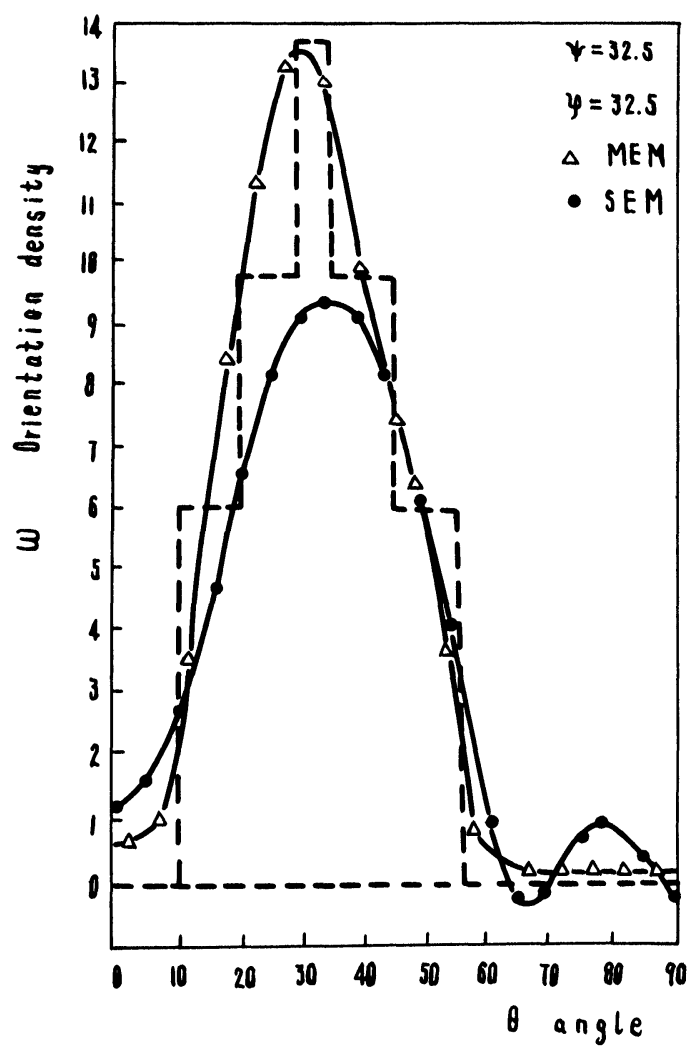

Figure 4 The orientation density vs. $\theta$ curves along $\psi=32.5^{\circ}$ and $\varphi=32.5^{\circ}$ calculated by the series expansion method (SEM) and by the MEM respectively. (The dash lines are originally assigned.)

Table 1 The odd term coefficients $W_{l m n}$ of the presumed sample

\begin{tabular}{|c|c|c|c|c|c|c|c|c|c|c|}
\hline$l$ & $n \quad m$ & 0 & 2 & 4 & 6 & 8 & 10 & 12 & 14 & 16 \\
\hline 7 & 0 & 0.0000 & 0.0807 & 0.119 & -0.0235 & & & & & \\
\hline 9 & 0 & 0.0000 & -0.0371 & 0.106 & -0.0211 & 0.0061 & & & & \\
\hline 11 & 0 & 0.0000 & -0.0385 & 0.05 & -0.0257 & -0.0200 & -0.0061 & & & \\
\hline 13 & 0 & 0.0000 & -0.0419 & 0.010 & -0.0186 & -0.0286 & 0.0043 & -0.0070 & & \\
\hline 13 & 6 & 0.1860 & -0.0064 & 0.01 & 0.0022 & 0.0128 & $0.0197-$ & -0.0459 & & \\
\hline 15 & 0 & 0.0000 & -0.0630 & 0.002 & -0.0224 & -0.0370 & 0.0072 & $-0.0036-$ & -0.0049 & \\
\hline 15 & 6 & 0.1188 & 0.0182 & $0.04 C$ & -0.0060 & -0.0003 & 0.0164 & 0.0007 & 0.0045 & \\
\hline 17 & 0 & 0.0000 & -0.0036 & -0.019 & -0.0189 & -0.255 & 0.0038 & $-0.0092-$ & $-0.0091-$ & -0.0037 \\
\hline 17 & 6 & 0.1347 & 0.0061 & 0.015 & -0.0120 & -0.0072 & 0.0001 & 0.0242 & 0.0031 & 0.0256 \\
\hline
\end{tabular}




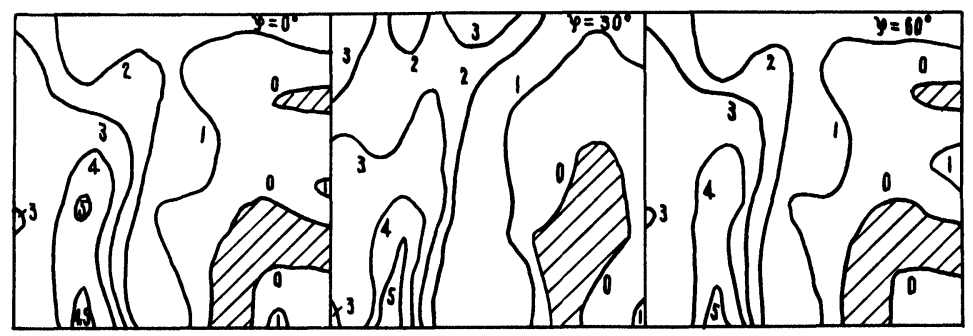

Figure 5 Some constant- $\varphi$ section of incomplete ODF of $\alpha$-Ti alloy sheet determined by the series expansion method.

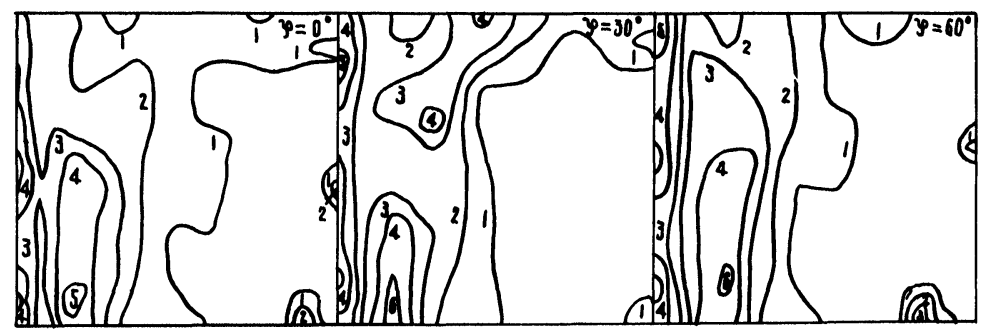

Figure 6 The ODF of the same sample reproduced by the MEM.

Figure 5 while Figure 6 is the result reproduced by the MEM from the same $W_{l m n}$ set as in Figure 5.

Though there is no significant difference between the two figures, yet the latter appears a sharper texture and does not exhibit negative region, which is more probably correct.

\section{CONCLUSION}

The MEM has been sucessfully applied to the ODF determination of hexagonal materials, and the experimental test confirms that the result of the MEM is a good approach to the complete ODF of the sample. No matter how distribution, smoothness and sharpness of the texture is, the method can be used without difficulty. 


\section{References}

Bunge, H. J. (1969) Mathematishe Methoden der Texturanalyse. Akademie Verlag, Berlin.

Bunge, H. J. and Esling, C. (1981) Proc. ICOTOM 6, Tokyo, Vol. 2, 1359-1372.

Dahms, M. and Bunge, H. J. (1988) Textures and Microstructures 10, 21-35.

Jaynes, E. T. (1957) Information theory and statistical mechanics. Phys. Review. 106, 620-630.

Matthies, S. (1981) General problems in reproducing the ODF from pole figures. Proc. ICOTOM 6, Tokyo, Vol. 1, 276-287.

Pospiech, J., Lücke, K. and Jura, J. (1981) Reproduction of the true ODF from pole figures and single orientation measurements by application of Gauss-type scattering models. Proc. ICOTOM 6, Vol. 2, 1390-1401.

Roe, R. J. (1965) J. Appl. phys. 36, 2024-2031.

Shannon, C. E. and Weaver, W. (1949) The mathematical theory of communication. University of Illinois Press. Urbana.

Van Houtte, P. (1983) The use of a quadratic form for the determination of non-negative texture function. Texture and Microstructures 6, 1-19.

Wang, F., Xu, J. and Liang, Z. (1987) Inverse pole figure determination according to the maximum entropy method. Proc. ICOTOM 8, Santa Fe.

Wei, W. (1985) J. Appl. Cryst. 18, 442-445.

Wei, W. (1986) Acmg physica simica. 35, 171-176. 